\title{
Sclerotinia sclerotiorum Thioredoxin Reductase Is Required for Oxidative Stress Tolerance, Virulence, and Sclerotial Development
}

\author{
Jinyi Zhang, Yabo Wang, Jiao Du, Zhiqiang Huang, Anfei Fang, Yuheng Yang, \\ Chaowei Bi, Ling Qing and Yang Yu* \\ College of Plant Protection, Southwest University, Chongqing, China
}

Sclerotinia sclerotiorum is a destructive ascomycete plant pathogen with worldwide distribution. Extensive research on different aspects of this pathogen's capability to cause disease will help to uncover clues about new ways to safely control Sclerotinia diseases. The thioredoxin (Trx) system consists of Trx and thioredoxin reductase (TrxR), which play critical roles in maintenance of cellular redox homeostasis. In this study, we

OPEN ACCESS

Edited by: Xin Li,

The University of British Columbia,

Canada

Reviewed by:

Mark Findlay Belmonte, University of Manitoba, Canada Hossein Borhan, Agriculture and Agri-Food Canada (AAFC), Canada

*Correspondence:

Yang Yu

zbyuyang@swu.edu.cn

Specialty section: This article was submitted to Plant Microbe Interactions,

a section of the journal

Frontiers in Microbiology

Received: 24 October 2018 Accepted: 28 January 2019 Published: 14 February 2019

Citation:

Zhang J, Wang Y, Du J, Huang Z, Fang $A$, Yang $Y, B i C$, Qing $L$ and $Y u Y$ (2019) Sclerotinia sclerotiorum

Thioredoxin Reductase Is Required for Oxidative Stress Tolerance,

Virulence, and Sclerotial Development.

Front. Microbiol. 10:233 doi: 10.3389/fmich.2019.00233 functionally characterized a gene encoding a TrxR (SsTrr1) in S. sclerotiorum. The amino acids of SsTrr1 exhibited high similarity with reported TrxRs in plant pathogens and targeted silencing of SsTrr1 lead to a decrease in TrxR activities of mycelium. SsTrr1 showed high expression levels during hyphae growth, and the levels decreased at the different stages of sclerotial development. SsTrr1 gene-silenced strains produced a smaller number of larger sclerotia on potato dextrose agar medium. The observations were consistent with the inhibitory effects on sclerotial development by the TrxR inhibitor, anrunofin. The expression of SsTrr1 showed a dramatic increase under the oxidative stress and the hyphal growth of gene-silenced strains showed more sensitivity to $\mathrm{H}_{2} \mathrm{O}_{2}$. SsTrr1 gene-silenced strains also showed impaired virulence in different hosts. Taken together, our results suggest that SsTrr1 encodes a TrxR that is of great important for oxidative stress tolerance, virulence, and sclerotial development of S. sclerotiorum.

Keywords: Sclerotinia sclerotiorum, thioredoxin reductase, oxidative stress, sclerotia, virulence, gene silencing

\section{INTRODUCTION}

Sclerotinia sclerotiorum is an ascomycete plant pathogen with a worldwide distribution (Bolton et al., 2006). This fungus infects more than 400 known plants and is the causal agent of stem rot in oilseed rape. S. sclerotiorum produces sclerotia, which are hard, asexual, resting structures. As melanized hyphal aggregates, sclerotia can survive for years in soil and play an important role in the disease cycle (Bolton et al., 2006; Erental et al., 2008). Sclerotia may germinate carpogenically to produce millions of airborne ascospores, which are the primary sources of inocula in most Sclerotinia diseases. Under certain conditions, sclerotia also germinate myceliogenically to produce hyphae, which can directly infect the hosts' stem or leaves (Schwartz and Steadman, 1978; Bardin and Huang, 2001).

Reactive oxygen species (ROS), including superoxide anion $\left(\mathrm{O}_{2}^{-}\right)$, hydrogen peroxide $\left(\mathrm{H}_{2} \mathrm{O}_{2}\right)$, and the hydroxyl radical $(\cdot \mathrm{OH})$ play important roles as secondary messengers in many intracellular signaling pathways (Mittler et al., 2011; Ray et al., 2012). However, high ROS concentrations can 
lead to DNA damage, protein inactivation and fragmentation, and lipid peroxidation (Aguirre et al., 2006). In plant-microbe interactions, ROS works as part of a defense mechanism and is a characteristic feature of the hypersensitive response (HR) (Lamb and Dixon, 1997). To detoxify ROS efficiently, cells usually use complex antioxidant responses, which mainly include superoxide dismutases, catalases, peroxidases, glutathione peroxidases, peroxiredoxins, and thioredoxins (Trxs) (Pomposiello et al., 2001; Aguirre et al., 2006).

The Trx system is ubiquitous from eukaryotes to archaea and plays a basic role in the maintenance of the redox environment in cells (Arnér and Holmgren, 2000). The Trx system is composed of $\operatorname{Trx}$, thioredoxin reductase ( $\operatorname{TrxR}$ ), and nicotinamide adenine dinucleotide phosphate (NADPH) (Holmgren, 1989). Trx contains a dithiol/disulfide active site (CGPC) and works as a major cellular disulfide reductase. Using NADPH as an electron donor, TrxR catalyzes the reduction of the active disulfide site in oxidized $\operatorname{TrxR}, \operatorname{Trx}-\mathrm{S}_{2}$, to a dithiol in reduced TrxR, Trx-(SH) 2 (Arnér and Holmgren, 2000). Reduced Trx directly reduces the disulfide in target proteins, and this process is required for several intracellular processes (Thön et al., 2007). In addition to Trx, TrxR also has other substrates, such as the glutaredoxin-like protein, $\mathrm{NrdH}$, in Escherichia coli (Jordan et al., 1997).

TrxR are homodimeric flavoenzymes that belong to a larger family of pyridine nucleotide-disulfide oxidoreductases. They contain an active redox disulfide and binding sites for flavin adenine dinucleotide (FAD) and NADPH in each subunit (Thön et al., 2007). TrxR can be divided into two classes according to the molecular weight (Ghisla and Massey, 1989). High molecular weight TrxR is present in higher eukaryotes and has a molecular weight of $55-58 \mathrm{kDa}$, while low molecular weight TrxR (homodimers of $35-36 \mathrm{kDa}$ subunits) is present in prokaryotes, archaea, plants, and fungi (Thön et al., 2007).

Recently, some fungal genes that encode TrxR have been cloned and functionally analyzed. Two TrxRs (Trr1 and 2) that have cytoplasmic and mitochondrial locations, respectively, were characterized in Saccharomyces cerevisiae (Pearson and Merrill, 1998; Pedrajas et al., 1999). An S. cerevisiae strain without trr2 showed more sensitivity to $\mathrm{H}_{2} \mathrm{O}_{2}$ (Pedrajas et al., 1999). Beauveria bassiana, a filamentous fungal insect pathogen, also contains two TrxR genes that play distinct roles in the redox system and host infection (Zhang et al., 2016). Some evidence has shown that the TrxRs are required for pathogenic activity in fungal plant pathogens. A loss of TrxR in Magnaporthe oryzae resulted in strains that failed to produce spreading necrotic lesions on the leaf surface (Fernandez and Wilson, 2014). The targeted deletion of TrxR in Alternaria alternata led to strains that were defective in $\mathrm{H}_{2} \mathrm{O}_{2}$ detoxification and induced smaller lesions on citrus leaves (Ma et al., 2018). In Botrytis cinerea, a fungus closely related to S. sclerotiorum, deletion of the TrxR-encoding gene, trrl, impaired fungal virulence and antioxidant capabilities (Viefhues et al., 2014). However, the role of TrxR in the development and pathogenicity of S. sclerotiorum is still unclear.

In this study, a gene encoding a putative TrxR has been identified, and its function in the sclerotial development and pathogenicity of $S$. sclerotiorum were characterized. The findings could help to advance our understanding of the role of TrxR in fungal plant pathogens and the molecular mechanisms that are involved in the sclerotial development and pathogenicity of S. sclerotiorum.

\section{MATERIALS AND METHODS}

\section{Fungal Strains and Culture Conditions}

The S. sclerotiorum isolate “1980" (Godoy et al., 1990) was used as the wild-type strain in this study. Strains were routinely cultured on potato dextrose agar (PDA) (Difco Laboratories, Detroit) at $20^{\circ} \mathrm{C}$. Transformants were cultured on PDA supplemented with hygromycin B at $100 \mu \mathrm{g} / \mathrm{mL}$ (Calbiochem, Riverside, CA, United States). The effects of auranofin (MedChem Express, Princeton, NJ, United States) on hyphae growth and sclerotial development were by adding a range of concentrations of auranofin $(0-62.5 \mu \mathrm{M})$ to the PDA medium.

\section{Vector Construction and Transform}

An SsTrr1 gene-silencing vector was constructed based on plasmid pCIT (Yu et al., 2012). The primer pairs SiTrr1ClaI (CGCATCGATTCAGCTCGCAGACTCGGTCT)/SiTrr1EcoRV (CGCGATATCTCGTTCCGGGCTTGGTTAC) and SiTrr1 BamHI (CGCGGATCCTCAGCTCGCAGACTCGGTCT)/SiTrr1 PstI (CGCCTGCAGTCGTTCCGGGCTTGGTTAC) were designed according to SsTrr1 cDNA sequences and then used to amplify the sense and antisense fragments of SsTrr1, respectively. The sense and antisense fragments were successfully inserted into the corresponding multiple cloning sites of the pCIT vector. A hygromycin resistance gene was then inserted into the $\mathrm{Xba}$ I site of the newly constructed vector to create the SsTrr1 RNA silencing vector pSiTrr1. The vector was then linearized with Xho I and used to transform the wild-type protoplasts of S. sclerotiorum according to the method used by Rollins (2003).

\section{Nucleic Acid Manipulation and Real-Time RT-PCR}

To assay the expression levels of SsTrr1 transcripts in different stages of sclerotial development of $S$. sclerotiorum, the wild-type strain was cultured on cellophane over PDA, and mycelia were harvested at 2 days post-inoculation (dpi) (hyphae), 3 dpi (initial sclerotia), 5 dpi (developing sclerotia), and 8 dpi (mature sclerotia). The mature sclerotia were cultured on the surface of moist sand at $16^{\circ} \mathrm{C}$ and collected once the stipe initials appeared. To evaluate the expression levels of SsTrr1 transcripts in different transforms containing pSiTrr1, the wild-type strains and the transforms were cultured on PDA for 3 days. To analyze the expression levels of SsTrr1 under oxidative stress conditions, the wild-type strain was cultured on PDB for 1 day, and then the culture was treated with $10 \mathrm{mM} \mathrm{H}_{2} \mathrm{O}_{2}$ for 1 day. The RNA products in different samples were extracted with a Trizol reagent (TianGen, Dalian, China). First-strand cDNA synthesis was performed using a ReventAid ${ }^{\text {TM }}$ First Strand cDNA Synthesis Kit (MBI Fermentas, Flamborough, 
ON, Canada). The relative expression levels of SsTrr1 were obtained with real-time reverse-transcriptase polymerase chain reaction (RT-PCR) using a CFX96 ${ }^{\mathrm{TM}}$ Real-time System (BioRad, Hercules, CA, United States). Real-time RT-PCR assays were performed according to $\mathrm{Yu}$ et al. (2012) with primer pair RT-SsTrr1fp (AGAATTTCCCTGGTTTCCCTAA)/RTSsTrr1rp (GTGTTCTGTCTTGTCATCCCATT), which was designed based on the cDNA of SsTrr1. The $\beta$-tubulin gene tub1 (SS1G_04652) was used as an internal control and amplified with the primer pair RT-tubfp (GTGAGGCT GAGGGCTGTGA)/RT-tubrp (CCTTTGGCGAT GGGACG).

\section{Pathogenicity Assay}

Pathogenicity assays were conducted on Arabidopsis thaliana col-0 and Nicotina benthamiana according to Yu et al. (2017) with slight modification. All plants were grown in a greenhouse at $25^{\circ} \mathrm{C}$ under a 16 -h light/8-h dark cycle. The plants or leaves were inoculated with mycelium agar plugs (6 $\mathrm{mm}$ in diameter) obtained from the edges of the growth colony of the wildtype strain and SsTrr1 gene-silenced strains. Photographs were taken at 24 and 48 hpi for $N$. benthamiana and A. thaliana, respectively. The experiment was repeated at least three times, and each strain was evaluated with at least five plants with three leaves (15 leaves total).

\section{Oxidative Stress Treatment}

In order to test the effects of oxidative stress on hyphal growth, the wild-type strain and SsTrr1 gene-silenced strains were cultured on PDA medium and PDA with $\mathrm{H}_{2} \mathrm{O}_{2}(5,10 \mathrm{mM})$. The colony diameters were then measured at $36 \mathrm{~h}$ to determine the inhibition of hyphal growth. Each experiment was repeated at least three times.

\section{Thioredoxin Reductase Activity Measurements}

The wild-type strain and SsTrr1 gene-silenced strains were cultured on PDA medium for 3 days. The total proteins for each strain were then extracted and used for the measurement of TrxR activities using a 5,5'-dithiobis-(2-nitrobenzoic acid) (DTNB) assay with assay kits (Solarbio, Beijing, China). Absorbance values were monitored at $412 \mathrm{~nm}$. TrxR activity levels were expressed as $\mathrm{U} / \mathrm{g}$ mycelium. The unit $\mathrm{U} / \mathrm{g}$ refers to the amount of TrxR in a 1 -g sample that catalytic reduction of $1 \mu$ mol DTNB per minute.

\section{RESULTS}

\section{Auranofin Inhibits the Hyphal Growth and Sclerotial Development of S. sclerotiorum}

In order to determine whether TrxR activity is involved in the development of S. sclerotiorum, the effects of the TrxR inhibitor auranofin on the hyphal growth and sclerotial formation were examined. The results showed that hyphal elongation was inhibited in the presence of auranofin in PDA medium (50\% effective concentration $\left[\mathrm{EC}_{50}\right]=4.4 \mu \mathrm{M}$ ) (Figure 1A). The number of sclerotia was negatively correlated with increasing concentrations of auranofin (Figure 1B), suggesting that auranofin inhibited S. sclerotiorum sclerotial formation. These results reveal the inhibitory effect of auranofin on S. sclerotiorum and support the possibility that TrxR is required for the hyphal growth and sclerotial development of this fungus.

\section{Characterization of Thioredoxin Reductase Gene in S. sclerotiorum}

In order to explore the roles of TrxR in the development and pathogenicity of S. sclerotiorum, one candidate TrxR-encoded gene (SS1G_05899) was identified using the genome sequence of S. sclerotiorum (Amselem et al., 2011). The gene comprises five exons encoding a 346-amino-acid polypeptide, which contain a TRX reductase domain at amino acid positions $\mathrm{K}^{4}$ to $\mathrm{L}^{306}$ $(E$-value $=2.18 e-145)$ according to a Conserved Domain Database (CDD) analysis (Marchler-Bauer et al., 2017). The sequence alignment demonstrated that the protein exhibited greater similarity with B. cinerea BcTrr1 (97\% identity and 100\%

A

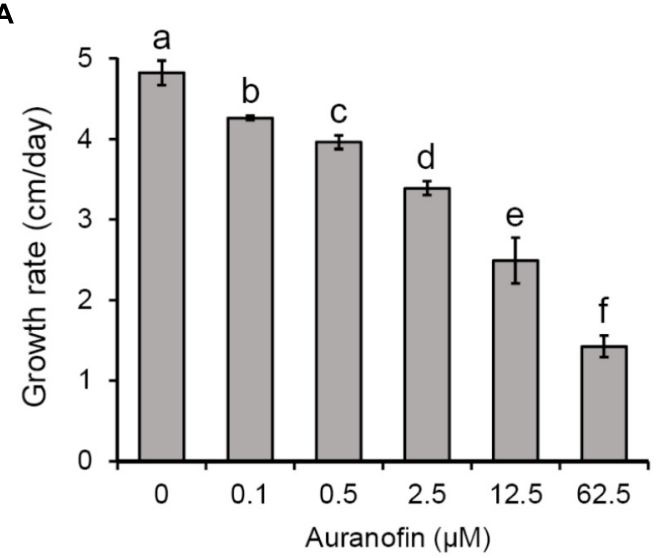

B

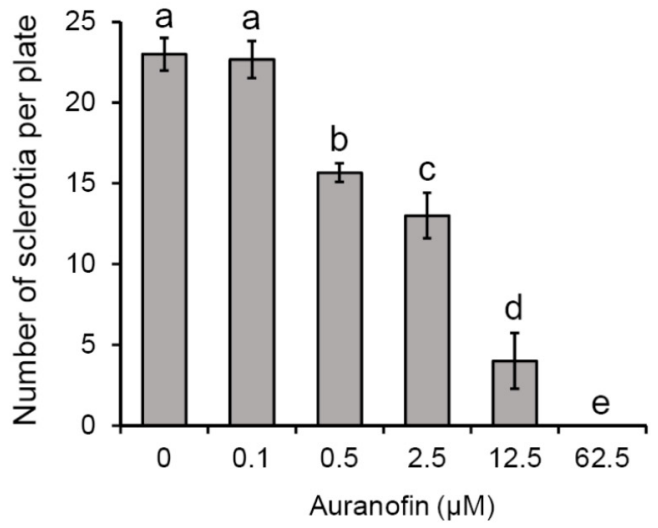

FIGURE 1 | Effects of auranofin on S. sclerotiorum colony growth and sclerotial formation. (A) Colony growth of S. sclerotiorum on PDA medium with auranofin at concentrations of 0-62.5 $\mu \mathrm{M}$. (B) Number of sclerotia on PDA medium with auranofin. Bars indicate standard deviation. Statistical significance was determined by one-way ANOVA and post hoc Tukey's tests. Different letters in the graph indicate statistical differences $(P<0.05)$. 
query coverage) (Viefhues et al., 2014), M. grisea MgTRR1 (82\% identity and 96\% query coverage) (Fernandez and Wilson, 2014), S. cerevisiae ScTRR1 (67\% identity and 94\% query coverage), and ScTRR2 (66\% identity and 99\% query coverage) (Pearson and Merrill, 1998; Pedrajas et al., 1999) (Figure 2). Thus, the gene was named SsTrr1 (S. sclerotiorum TrxR 1). To provide initial insight into the role of TrxR in fungal development, a real-time RT-PCR analysis was used to measure the abundance of SsTrr1 mRNA in different growth stages of S. sclerotiorum. As shown in Figure 3, SsTrr1 exhibited constitutive expression at different sclerotia development stages. However, the expression levels in the hyphae were twice that seen during the sclerotial development.

\section{Functional Analysis of SsTrr1 in S. sclerotiorum}

The gene-silenced vector pSiTrr1 was constructed as descripted in the section "Materials and Methods" in order to functionally analyze SsTrr1 in S. sclerotiorum. The vector was used to transform the wild-type strain 1980 as described by Rollins (2003), and several transforms were obtained. SsTrr1 expression levels in several different randomly selected transformants were determined with real-time RT-PCR, and SiTrr1-54 and SiTrr159 showed a dramatic decrease in the abundances of SsTrr1 mRNA (Figure 4). Thus, these two strains were chosen for further study. The wild-type strain and SsTrr1 gene-silenced strains were cultured for 3 days, and the TrxR activity in the mycelium was then determined using a DTNB assay. The results showed that the activities of TrxR in SiTrr1-54 and SiTrr159 were significantly decreased in comparison with that in the wild-type strain (Figure 5). This suggests that the inhibition of the SsTrr1 expression levels leads to reduced TrxR activities in S. sclerotiorum.

\section{SsTrr1 in Relation to Sclerotia Development}

When cultured on PDA plates, the two SsTrr1 gene-silenced strains showed similar morphology of the hyphal branch to that of the wild-type strain (data not shown). However, the SsTrr1 gene-silenced strains produced less of the slightly bigger sclerotia that formed in a random manner in the
SsTrr1

BcTrr1

MgTRR1

SCTRR1

SCTRR2

SsTrr1

BcTrr1

MgTRR1

SCTRR1

SCTRR2

SsTrr1

BcTrr1

MgTRR1

SCTRR1

SCTRR2

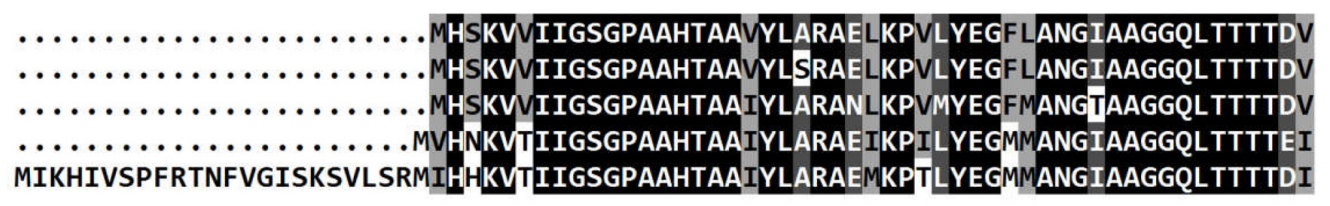

MIKHIVSPFRTNFVGISKSVLSRMIHHKVTIIGSGPAAHTAAIYLARAEMKPTLYEGMMANGIAAGGQLTTTTDI
ENFPGFPKGIGGQELMDNMRAQSERFGTQIITETVAKVDLSKRPFKYWTEWDDKTE.HTADSIIIATGASARRLG ENFPGFPKGIGGQELMDNMRAQSERFGTQIITETVAKVDLSKRPFKYWTEWDDKTE. HTADSIIIATGASARRLG ENFPGFPKGIGGQELMDAMREQSSRFETEIISETVAKVDLSSRPFKYCTEWSPEVM. HTADTLVIATGASARRLG ENFPGFPDGLTGSELMDRMREQSTKFGTEIITETVSKVDLSSKPFKLWTEFNEDAEPVTTDAIILATGASAKRMH ENFPGFPESLSGSELMERMRKQSAKFGTNIITETVSKVDLSSKPFRLWTEFNEDAEPVTTDAI ILATGASAKRMH

SsTrr1

LPGEEKYWQNGISACAVCDGAVPIFRNKPLVIGGGDSAAEEAMFLTKYGSHVTVLVRKDHLRASKTMAKRLLAN

FIGURE 2 | Clustal X alignment of the amino acid sequence of SsTrr1 with several reported TrxRs of different fungi, including M. oryzae TRR1 (EHA54395.1), B. cinerea BcTrr1 (XP_001560033.1), S. cerevisiae Trr1 (KZV12592.1), and Trr2 (KZV12592.1). Shading indicates sequence similarities of 100 (dark), 75 (medium), and $50 \%$ (light). Numbers mean the amino acid of the predicted polypeptide. 


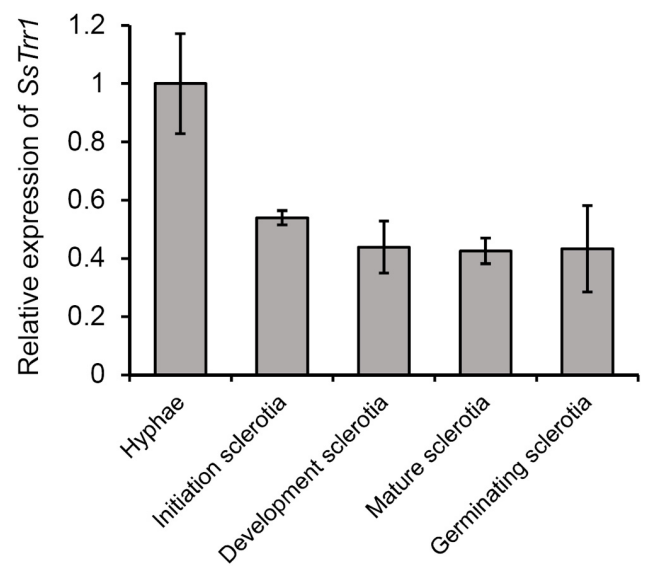

FIGURE 3 | Ss Trr1 transcript in different sclerotial development stages as determined by real-time reverse transcription-polymerase chain reaction (RT-PCR). The quantity of SsTrr1 cDNA was normalized to that of tub1 cDNA in extracts from each developmental stage. The abundance of cDNA in the mycelium sample was set as unity. Bars indicate standard error.

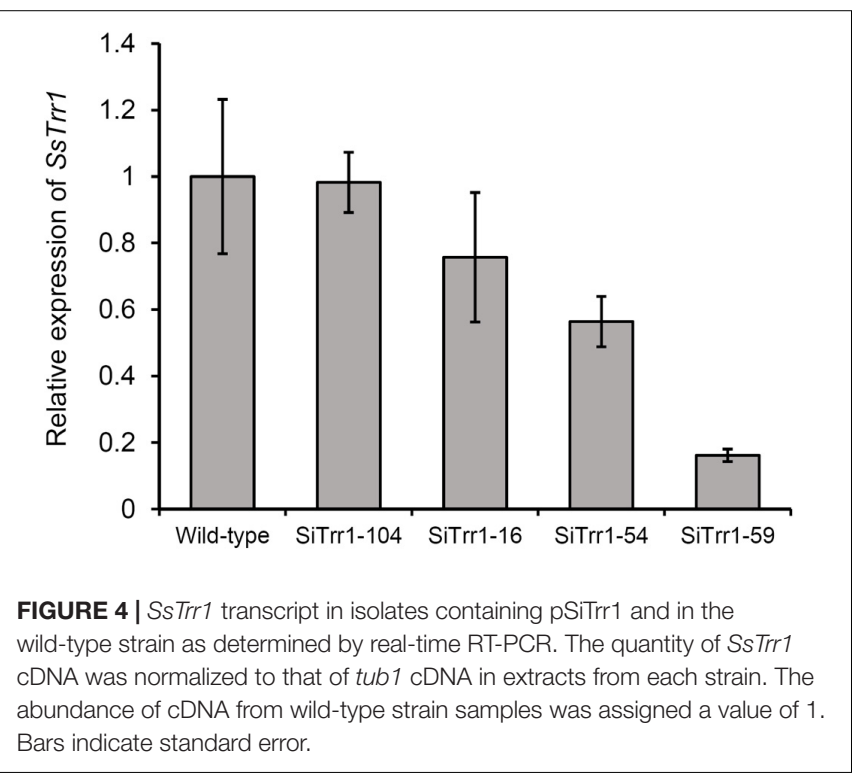

plates (Figures 6A,B). The average numbers and dry weights of the sclerotia produced by SiTrr1-59 per $9-\mathrm{cm}$ plate were approximately 74 and $110 \%$ of those produced by the wild-type strain, respectively (Figures 6C,D). The results were consistent with previous findings of sclerotial formation being inhibited by auranofin, suggesting that SsTrr1 is related to sclerotial development in S. sclerotiorum.

\section{SsTrr1 Related to Pathogenicity}

In order to determine the effects of SsTrr1 silencing on the pathogenicity of S. sclerotiorum, detached A. thaliana leaves were inoculated with mycelium plugs of SsTrr1 gene-silenced strains. As demonstrated in Figure 7A, SiTrr1-54 and SiTrr1-59 led to small lesions on the A. thaliana leaves compared to the wild-type

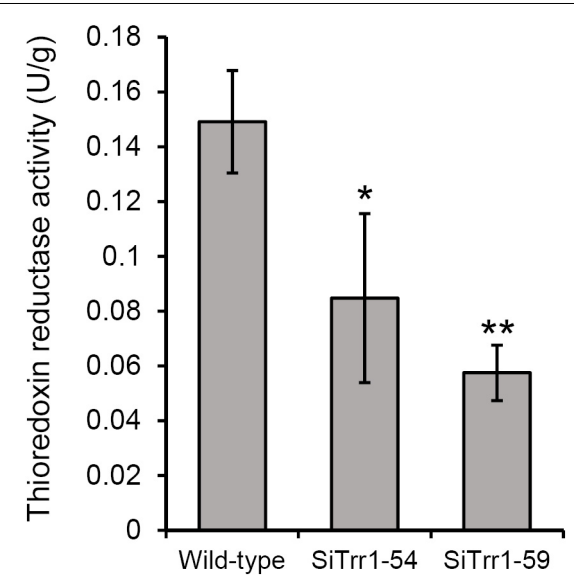

FIGURE 5 | Total TrxR activity quantified in the extracts of 3-day-old PDA cultures of SsTrr1 gene-silenced strains. Bars indicate standard deviation. Asterisks denote significant differences (one-way ANOVA). ${ }^{*} P<0.05$; $* * P<0.01$.

strain. The pathogenicity of the two gene-silenced strains was also tested on intact $N$. benthamiana plants, and smaller lesions were observed on the leaves (Figure 7B). These results indicate that SsTrr1 is required for the full virulence of S. sclerotiorum.

\section{SsTrr1 in Relation to Oxidative Stress Tolerance}

The relative SsTrr1 expression levels under oxidative stress were determined via real-time RT-PCR in order to extensively characterize the role of $S s T r r 1$ in response to oxidative stress in S. sclerotiorum. As shown Figure 8A, the SsTrr1 expression level increased sharply in hyphae that were treated with $10 \mathrm{mM}$ $\mathrm{H}_{2} \mathrm{O}_{2}$. The hyphal growth under oxidative stress between the wild-type and SsTrr1 gene-silenced strains was then compared. When growth on PDA plates was amended with $\mathrm{H}_{2} \mathrm{O}_{2}$, hyphal growth inhibition was significantly greater for SiTrr154 and SiTrr1-59 than the wild-type strain (Figure 8B). The results indicate that $S s T r r 1$ contributes to the oxidative stress tolerance in S. sclerotiorum.

\section{DISCUSSION}

In this study, the gene SsTrr1, which encodes a TrxR in S. sclerotiorum, was cloned and functionally analyzed. SsTrr1 was shown to have an effect on the oxidative stress tolerance, sclerotial development, and pathogenicity of S. sclerotiorum.

Evidence has shown that the numbers of TrxRs vary among different fungi. In S. cerevisiae, two TrxRs (Trr1 and 2) were identified and were shown to be located in the cytoplasm and mitochondria, respectively (Pearson and Merrill, 1998; Pedrajas et al., 1999). The filamentous fungal insect pathogen B. bassiana also contains two TrxRs that play distinct roles in the redox system and host infection (Zhang et al., 2016). However, only one TrxR was identified in fungal plant pathogens, including B. cinerea, M. oryzae, and A. alternata, and a cytoplasmic location 


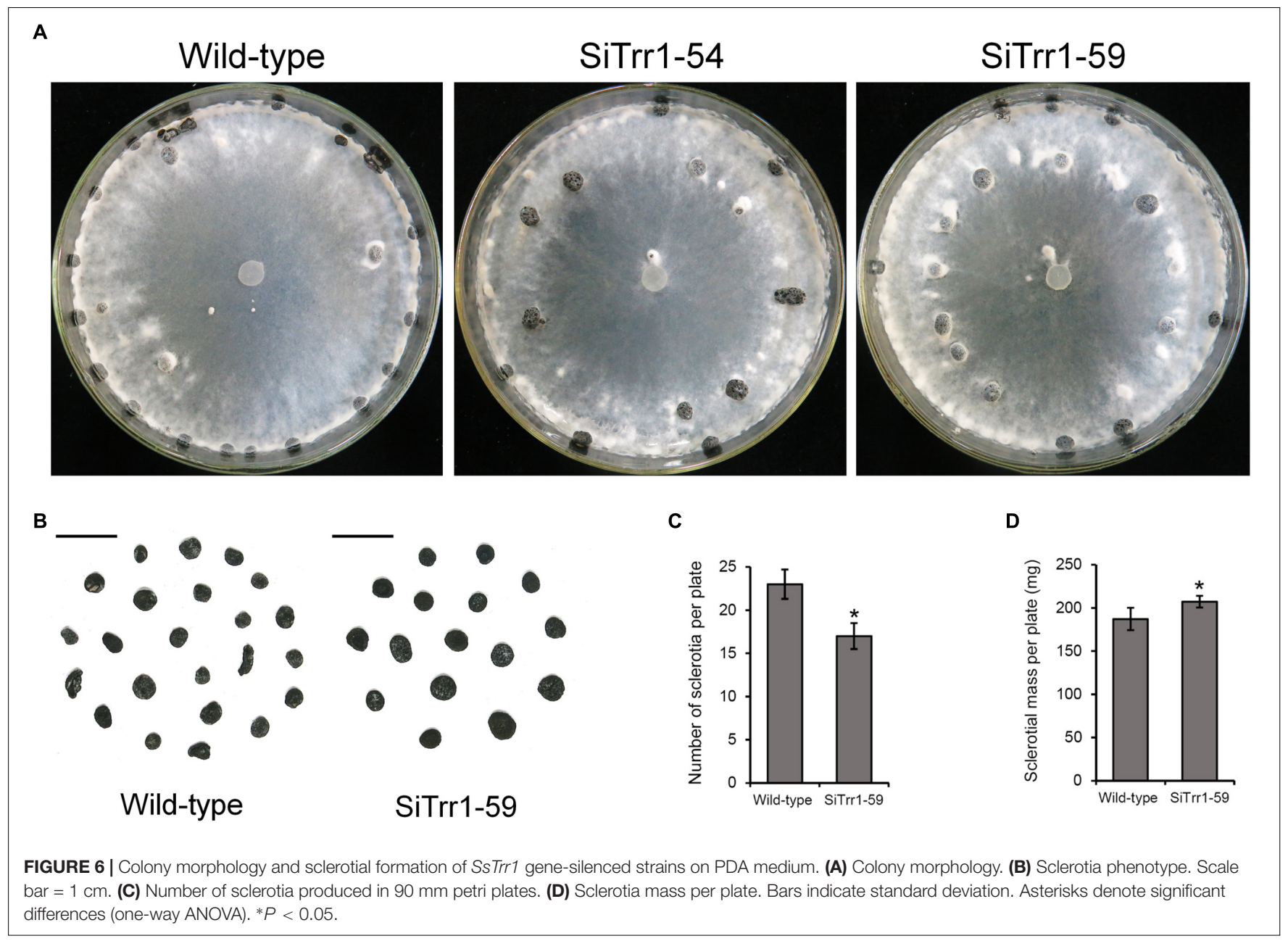

was demonstrated in these fungi (Fernandez and Wilson, 2014; Viefhues et al., 2014; Ma et al., 2018). BLASTP searches indicated that S. sclerotiorum contains only one TrxR-encoding gene, and this number is consistency with other fungal plant pathogens. Since there is a lack of effective fluorescent protein labeling methods, the subcellular localization of TrxR in S. sclerotiorum was predicted using ProtCom 9.0 servers $^{1}$, and the results showed that SsTrr1 is most likely localized in the cytoplasm.

Some genes that are related to ROS modulation in S. sclerotiorum have been discussed. Functional loss of a $\mathrm{Cu} / \mathrm{Zn}$ superoxide dismutase in $S$. sclerotiorum resulted in an increase in sensitivity to oxidative stress in culture (Veluchamy et al., 2012; Xu and Chen, 2013). However, deletion of the catalase, SCAT1, in S. sclerotiorum led to an increase in tolerance to $\mathrm{H}_{2} \mathrm{O}_{2}$, indicating that SCAT1 is not essential for $\mathrm{H}_{2} \mathrm{O}_{2}$ degradation in vitro (Yarden et al., 2014). In recent years, several fungal TrxR-encoding genes have been shown to play critical roles in oxidative stress responses (Fernandez and Wilson, 2014; Viefhues et al., 2014; Ma et al., 2018). In this study, SsTrr1 showed a sharp increase in expression under oxidative stress conditions,

${ }^{1}$ http://www.softberry.com/ and the gene-silenced strains exhibited sensitivity to $\mathrm{H}_{2} \mathrm{O}_{2}$, suggesting a conserved function for TrxR in oxidative stress in fungi.

The generation of ROS has been recorded as one of the earliest resistance responses for plants against fungal pathogens (Bolwell et al., 1995). ROS detoxification and tolerance are critical for S. sclerotiorum hyphae to infect host plants successfully (Kim et al., 2011; Williams et al., 2011; Yarden et al., 2014). Previous evidence has shown that these genes, which play critical roles in the detoxification and tolerance of ROS, are essential for S. sclerotiorum pathogenesis (Veluchamy et al., 2012; Xu and Chen, 2013; Yu et al., 2015). McLoughlin et al. (2018) reported that foliar applications of dsRNAtargeted SsTrr1 reduced S. sclerotiorum infection in B. napus. In this study, we found that SsTrr1 gene-silenced strains exhibited attenuated virulence in different hosts. SiTrr1-59 with a lower expression level of SsTrr1 led to smaller lesions. These data further indicted that TrxR is critical for the successful infection of this fungus. The role of TrxR in $S$. sclerotiorum virulence is consistent with those of fungal plant pathogens such as B. cinerea (Viefhues et al., 2014), A. alternata (Ma et al., 2018), and M. oryzae (Fernandez and Wilson, 2014), in addition to the fungal insect pathogen 
A

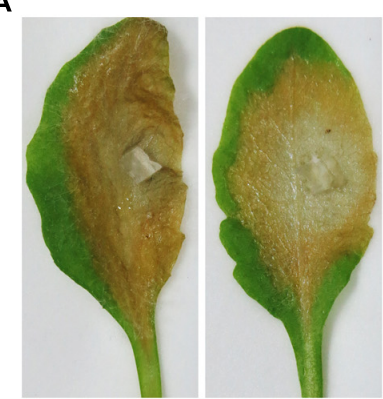

Wild-type

B

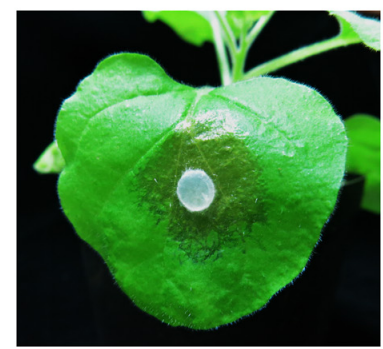

Wild-type

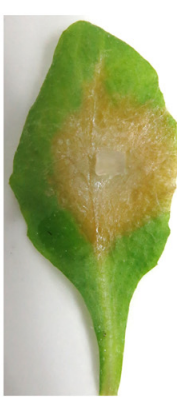

SiTrr1-54

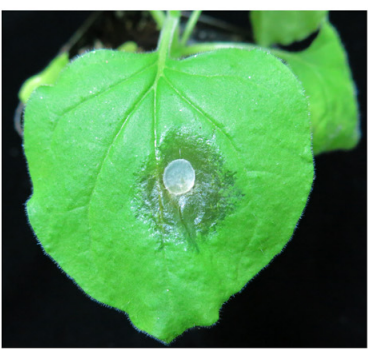

SiTrr1-54
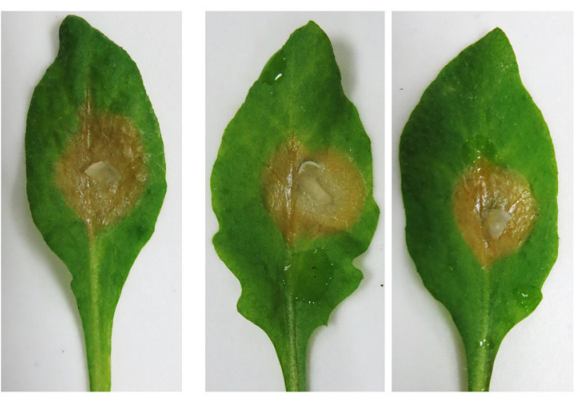

SiTrr1-59

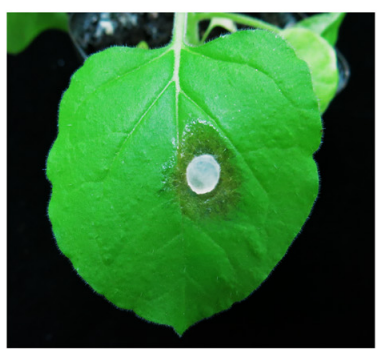

SiTrr1-59

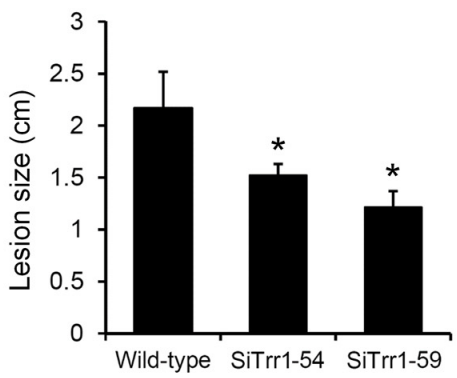

FIGURE 7 | Pathogenicity analysis of SsTrr1 gene-silenced strains. Detached leaves of $A$. thaliana (A) and intact $N$. benthamiana plants (B) were inoculated with PDA plugs colonized with the wild-type strain, SiTrr1-54 and SiTrr1-59. Lesion size were measured at 24 and 48 hpi for N. benthamiana and A. thaliana, respectively. Error bars indicate standard deviation. Statistical significance is indicated in the graph (one-way ANOVA). ${ }^{*} P<0.05$.
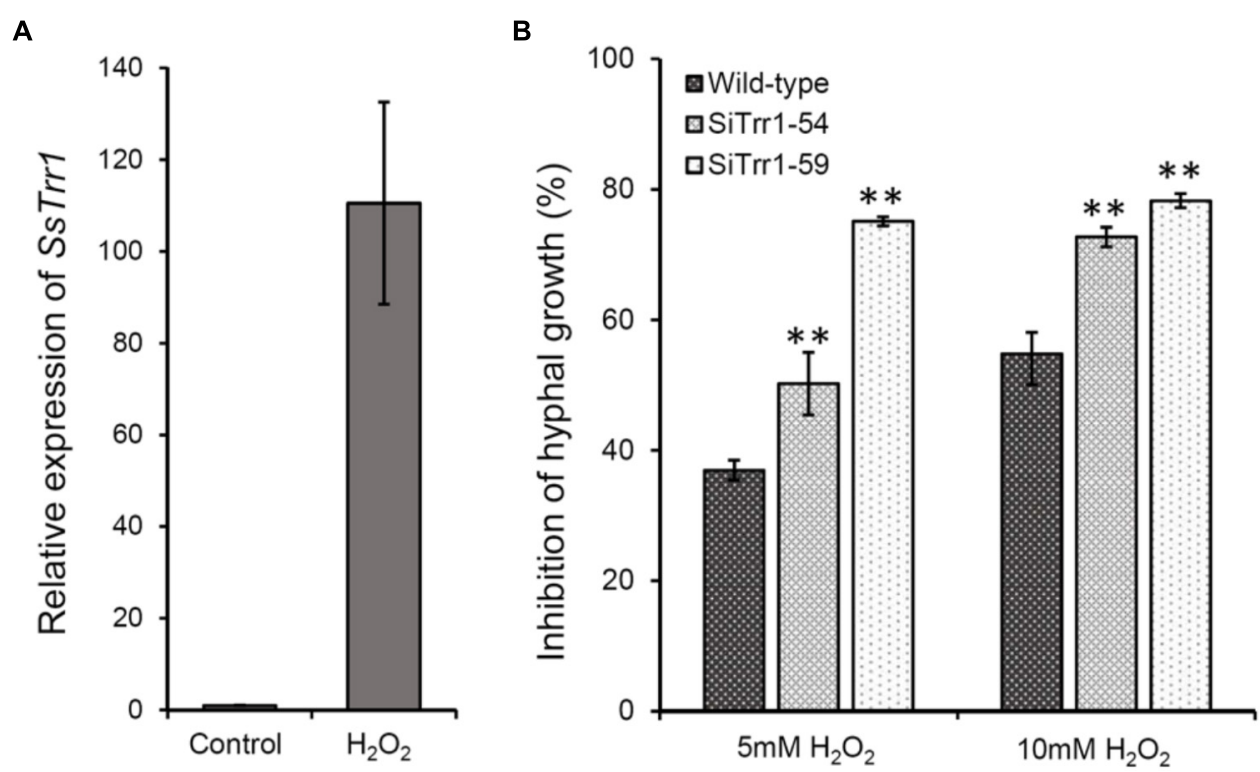

FIGURE 8 | Functional analysis of SsTrr1 in oxidative stress response. (A) SsTrr1 transcript in hyphae treated with 10 mM $\mathrm{H}_{2} \mathrm{O}_{2}$. Total SsSvf1 cDNA abundance in the samples was normalized to using tub1 gene as a control. The relative expression of SsTrr1 in the untreated strain was set as 1. Bars indicate standard error. (B) Percent growth inhibition of wild-type strain and SsTrr1 gene-silenced strains on PDA medium with 5 and $10 \mathrm{mM} \mathrm{H}_{2} \mathrm{O}_{2}$. Bars indicate standard deviation. Asterisks denote significant differences (one-way ANOVA). ${ }^{* *} P<0.01$. 
B. bassiana (Zhang et al., 2016). It is suggested that the absence of a component of the Trx system may lead to a disturbance of fungal redox balance, which is critical for fungal infection and colonization.

Sclerotia are important dormant bodies for many fungal plant pathogens in Ascomycota and Basidiomycota, including S. sclerotiorum, B. cinerea, Rhizoctonia solani, and Verticillium dahliae. Sclerotial development in S. sclerotiorum is a complicated biological process and can be divided into three distinguishable stages: (1) initiation, (2) development, and (3) maturation (Willetts and Bullock, 1992). Sclerotial formation is affected by several environmental signals, including nutrient limitation, light, $\mathrm{pH}$, and temperature, and is under the control of cyclic adenosine monophosphate (cAMP)/protein kinase A (PKA) and mitogen-activated protein kinase (MAPK) cellular signaling pathways (Chet and Henis, 1975; Chen et al., 2004; Jurick et al., 2004; Jurick and Rollins, 2007). Classical theory postulates that hyperoxidant states trigger microbial eukaryotic cell differentiation (Lara-Ortiz et al., 2003; Aguirre et al., 2005). Previous studies have also shown that sclerotial differentiation is associated with an increase in oxidative level, and oxidative stress promoted sclerotial metamorphosis (Georgiou et al., 2006; Papapostolou et al., 2014). Silencing of the NADPH oxidase genes SsNox1 and SsNox2 resulted in reduced ROS levels and limited sclerotial development of S. sclerotiorum (Kim et al., 2011). However, a $\mathrm{Cu} / \mathrm{Zn}$ superoxide dismutase gene-deletion mutant exhibited normal sclerotial formation ( $\mathrm{Xu}$ and Chen, 2013), indicating a complex role for ROS in the sclerotial development of S. sclerotiorum.

In this study, sclerotial development was suppressed by auranofin, which inhibited TrxR activity. Furthermore, targeted silencing of the TrxR-encoding gene SsTrr1 led to the production fewer of sclerotia. To our knowledge, this is the first report that TrxRs are required for fungal sclerotial development. However, the level of SsTrr1 expression exhibited a decrease when sclerotia began to form, which indicated a dynamic balance of TrxR activity is critical for sclerotial development. Since TrxR is critical and consists of Trx systems that act against oxidative stress, we hypothesized that TrxR impacts sclerotial development via intracellular redox-level regulation. S. sclerotiorum often

\section{REFERENCES}

Aguirre, J., Hansberg, W., and Navarro, R. (2006). Fungal responses to reactive oxygen species. Med. Mycol. 44(Suppl. 1), S101-S107. doi: 10.1080/ 13693780600900080

Aguirre, J., Ríos-Momberg, M., Hewitt, D., and Hansberg, W. (2005). Reactive oxygen species and development in microbial eukaryotes. Trends Microbiol. 13, 111-118. doi: 10.1016/j.tim.2005.01.007

Amselem, J., Cuomo, C. A., van Kan, J. A., Viaud, M., Benito, E. P., Couloux, A., et al. (2011). Genomic analysis of the necrotrophic fungal pathogens Sclerotinia sclerotiorum and Botrytis cinerea. PLoS Genet. 8:e1002230. doi: 10.1371/journal. pgen. 1002230

Arnér, E. S., and Holmgren, A. (2000). Physiological functions of thioredoxin and thioredoxin reductase. Eur. J. Biochem. 267, 6102-6109. doi: 10.1046/j.14321327.2000.01701.x

Bardin, S. D., and Huang, H. C. (2001). Research on biology and control of Sclerotinia diseases in canada. Can. J. Plant Pathol. 23, 88-98. doi: 10.1080/ 07060660109506914 produces a ring of sclerotia at the edge of the colony, mainly because of inhibition of polar elongation, staling compounds, and nutrient limitation (Chet and Henis, 1975; Jurick and Rollins, 2007). In this study, targeted silencing of SsTrr1 resulted in sclerotial formation in a random manner, and most of the sclerotia were located in the center of the plates. Since SsTrr1 gene-silenced strains showed normal hyphal growth, SsTrr1 silencing may lead to impaired sensing of staling compounds and nutrient limitation. It is interesting to note that catalase SCAT1 deletion strains also produced sclerotia in the center of the plates (Yarden et al., 2014), indicating that the maintenance of the redox status is indeed necessary for the sclerotial development of S. sclerotiorum. The connection between the substrates of TrxRs and the sclerotial development of S. sclerotiorum requires additional studies.

\section{AUTHOR CONTRIBUTIONS}

JZ, YW, JD, ZH, AF, YhY, CB, LQ, and YY conceived and designed the experiments, and contributed reagents, materials, and analysis tools. JZ and YY performed the experiments, analyzed the data, and wrote the manuscript. All authors read and approved the final manuscript.

\section{FUNDING}

This research was funded by National Natural Science Foundation of China (No. 31671973), National Key Research and Development Program of China (2018YFD0200900), Chongqing Research Program of Basic Research and Frontier Technology (No. cstc2017jcyjAX0096), Fundamental Research Funds for the Central Universities, China (Nos. XDJK2017A006 and XDJK2018AA004).

\section{ACKNOWLEDGMENTS}

We thank reviewers for their kind suggestions.

Bolton, M. D., Thomma, B. P., and Nelson, B. D. (2006). Sclerotinia sclerotiorum (Lib.) de Bary: biology and molecular traits of a cosmopolitan pathogen. Mol. Plant Pathol. 7, 1-16. doi: 10.1111/j.1364-3703.2005.00316.x

Bolwell, G. P., Butt, V. S., Davies, D. R., and Zimmerlin, A. (1995). The origin of the oxidative burst in plants. Free Radic. Res. 23, 517-532. doi: 10.3109/ 10715769509065273

Chen, C. B., Harel, A., Gorovoits, R., Yarden, O., and Dickman, M. B. (2004). MAPK regulation of sclerotial development in Sclerotinia sclerotiorum is linked with $\mathrm{pH}$ and cAMP sensing. Mol. Plant Microbe Interact. 17, 404-413. doi: 10.1094/MPMI.2004.17.4.404

Chet, I., and Henis, Y. (1975). Sclerotial morphogenesis in fungi. Annu. Rev. Phytopathol. 13, 169-192. doi: 10.1146/annurev.py.13.090175.001125

Erental, A., Dickman, M. B., and Yarden, O. (2008). Sclerotial development in Sclerotinia sclerotiorum: awakening molecular analysis of a "Dormant" structure. Fungal Bio. Rev. 22, 6-16. doi: 10.1016/j.fbr.2007. 10.001

Fernandez, J., and Wilson, R. A. (2014). Characterizing roles for the glutathione reductase, thioredoxin reductase and thioredoxin peroxidase-encoding genes 
of Magnaporthe oryzae during rice blast disease. PLoS One 9:e87300. doi: 10. 1371/journal.pone. 0087300

Georgiou, C. D., Patsoukis, N., Papapostolou, I., and Zervoudakis, G. (2006). Sclerotial metamorphosis in filamentous fungi is induced by oxidative stress. Integr. Comp. Biol. 46, 691-712. doi: 10.1093/icb/icj034

Ghisla, S., and Massey, V. (1989). "Mechanisms of flavoprotein-catalyzed reactions," in The Ejb Reviews, eds P. Christen and E. Hofman (Berlin: Springer), 29-45.

Godoy, G., Steadman, J. R., Dickman, M. B., and Dam, R. (1990). Use of mutants to demonstrate the role of oxalic acid in pathogenicity of Sclerotinia sclerotiorum on Phaseolus vulgaris. Physiol. Mol. Plant Pathol. 37, 179-191. doi: 10.1016/ 0885-5765(90)90010-U

Holmgren, A. (1989). Thioredoxin and glutaredoxin systems. J. Biol. Chem. 264, 13963-13966.

Jurick, W. M. II, Dickman, M. B., and Rollins, J. A. (2004). Characterization and functional analysis of a cAMP-dependent protein kinase a catalytic sub unit gene (pka1) in Sclerotinia sclerotiorum. Physiol. Mol. Plant Pathol. 64, 155-163. doi: 10.1016/j.pmpp.2004.07.004

Jurick, W. M. II, and Rollins, J. A. (2007). Deletion of the adenylate cyclase (SAC1) gene affects multiple developmental pathways and pathogenicity in Sclerotinia sclerotiorum. Fungal Genet. Biol. 44, 521-530. doi: 10.1016/j.fgb.2006.11.005

Jordan, A., Aslund, F., Pontis, E., Reichard, P., and Holmgren, A. (1997). Characterization of Escherichia coli $\mathrm{NrdH}$. a glutaredoxin-like protein with a thioredoxin-like activity profile. J. Biol. Chem. 272, 18044-18050. doi: 10.1074/ jbc.272.29.18044

Kim, H. J., Chen, C., Kabbage, M., and Dickman, M. B. (2011). Identification and characterization of Sclerotinia sclerotiorum NADPH oxidases. Appl. Environ. Microb. 77, 7721-7729. doi: 10.1128/AEM.05472-11

Lamb, C., and Dixon, R. A. (1997). The oxidative burst in plant disease resistance. Annu. Rev. Plant Physiol. 48, 251-275. doi: 10.1146/annurev.arplant.48.1.251

Lara-Ortiz, T., Riveros-Rosas, H., and Aguirre, J. (2003). Reactive oxygen species generated by microbial NADPH oxidase NoxA regulate sexual development in Aspergillus nidulans. Mol. Microbiol. 50, 1241-1255. doi: 10.1046/j.1365-2958. 2003.03800.x

Ma, H., Wang, M., Gai, Y., Fu, H., Zhang, B., Ruoxin, R., et al. (2018). Thioredoxin and glutaredoxin systems required for oxidative stress resistance, fungicide sensitivity, and virulence of Alternaria alternate. Appl. Environ. Microbiol. 84, e00086-e00118. doi: 10.1128/AEM.00086-18

Marchler-Bauer, A., Bo, Y., Han, L., He, J., Lanczycki, C. J., Lu, S., et al. (2017). CDD/SPARCLE: functional classification of proteins via subfamily domain architectures. Nucleic Acids Res. 45, D200-D203. doi: 10.1093/nar/gkw1129

McLoughlin, A. G., Wytinck, N., Walker, P. L., Girard, I. J., Rashid, K. Y., de Kievit, T., et al. (2018). Identification and application of exogenous dsRNA confers plant protection against Sclerotinia sclerotiorum and Botrytis cinerea. Sci. Rep. 8:7320. doi: 10.1038/s41598-018-25434-4

Mittler, R., Vanderauwera, S., Suzuki, N., Miller, G., Tognetti, V. B., Vandepoele, K., et al. (2011). ROS signaling: the new wave? Trends Plant Sci. 16, 300-309. doi: 10.1016/j.tplants.2011.03.007

Papapostolou, L., Sideri, M., and Georigou, C. D. (2014). Cell proliferating and differentiating role of $\mathrm{H}_{2} \mathrm{O}_{2}$ in Sclerotium rolfsii and Sclerotinia sclerotiorum. Microbiol. Res. 169, 527-532. doi: 10.1016/j.micres.2013.12.002

Pearson, G. D., and Merrill, G. F. (1998). Deletion of the Saccharomyces cerevisiae TRR1 gene encoding thioredoxin reductase inhibits p53-dependent reporter gene expression. J. Biol. Chem. 273, 5431-5434. doi: 10.1074/jbc.273.10.5431

Pedrajas, J. R., Kosmidou, E., Miranda-Vizuete, A., Gustafsson, J. A., Wright, A. P., and Spyrou, G. (1999). Identification and functional characterization of a novel mitochondrial thioredoxin system in Saccharomyces cerevisiae. J. Biol. Chem. 274, 6366-6373. doi: 10.1074/jbc.274.10.6366

Pomposiello, P. J., Bennik, M. H., and Demple, B. (2001). Genome-wide transcriptional profiling of the Escherichia coli responses to superoxide stress and sodium salicylate. J. Bacteriol. 183, 3890-3902. doi: 10.1128/JB.183.13. 3890-3902.2001
Ray, P. D., Huang, B., and Tsuji, Y. (2012). Reactive oxygen species (ROS) homeostasis and redox regulation in cellular signaling. Cell. Signal. 24, 981-990. doi: 10.1016/j.cellsig.2012.01.008

Rollins, J. A. (2003). The Sclerotinia sclerotiorum pacl gene is required for sclerotial development and virulence. Mol. Plant Microbe Interact. 16, 785-795. doi: 10.1094/MPMI.2003.16.9.785

Schwartz, H. F., and Steadman, J. R. (1978). Factors affecting sclerotium populations of, and apothecium production by, Sclerotinia sclerotiorum. Phytopathology 68, 383-388. doi: 10.1094/Phyto-68-383

Thön, M., Al-Abdallah, Q., Hortschansky, P., and Brakhage, A. A. (2007). The thioredoxin system of the filamentous fungus Aspergillus nidulans impact on development and oxidative stress response. J. Biol. Chem. 282, 27259-27269. doi: $10.1074 /$ jbc.M704298200

Veluchamy, S., Williams, B., Kim, K., and Dickman, M. B. (2012). The CuZn superoxide dismutase from Sclerotinia sclerotiorum is involved with oxidative stress tolerance, virulence, and oxalate production. Physiol. Mol. Plant Pathol. 78, 14-23. doi: 10.1016/j.pmpp.2011.12.005

Viefhues, A., Heller, J., Temme, N., and Tudzynski, P. (2014). Redox systems in Botrytis cinerea: impact on development and virulence. Mol. Plant Microbe Interact. 27, 858-874. doi: 10.1094/MPMI-01-14-0012-R

Willetts, H. J., and Bullock, S. (1992). Developmental biology of sclerotia. Mycol. Res. 96, 801-816. doi: 10.1016/S0953-7562(09)81027-7

Williams, B., Kabbage, M., Kim, H. J., Britt, R., and Dickman, M. B. (2011). Tipping the balance: Sclerotinia sclerotiorum secreted oxalic acid suppresses host defenses by manipulating the host redox environment. PLoS Pathog. 7:e1002107. doi: 10.1371/journal.ppat.1002107

$\mathrm{Xu}, \mathrm{L}$., and Chen, W. (2013). Random T-DNA mutagenesis identifies a $\mathrm{Cu} / \mathrm{Zn}$ superoxide dismutase gene as a virulence factor of Sclerotinia sclerotiorum. Mol. Plant Microbe Interact. 26, 431-441. doi: 10.1094/MPMI-07-12-0177-R

Yarden, O., Veluchamy, S., Dickman, M. B., and Kabbage, M. (2014). Sclerotinia sclerotiorum catalase SCAT1 affects oxidative stress tolerance, regulates ergosterol levels and controls pathogenic development. Physiol. Mol. Plant Pathol. 85, 34-41. doi: 10.1016/j.pmpp.2013.12.001

Yu, Y., Jiang, D., Xie, J., Cheng, J., Li, G., Yi, X., et al. (2012). Ss-Sl2, a novel cell wall protein with PAN modules, is essential for sclerotial development and cellular integrity of the plant fungal pathogen Sclerotinia sclerotiorum. PLoS One 7:e34962. doi: 10.1371/journal.pone.0034962

Yu, Y., Xiao, J., Yang, Y., Bi, C., Qing, L., and Tan, W. (2015). Ss-Bil encodes a putative BAX inhibitor-1 protein that is required for full virulence of Sclerotinia sclerotiorum. Physiol. Mol. Plant Pathol. 90, 115-122. doi: 10.1016/j.pmpp.2015. 04.005

Yu, Y., Xiao, J., Zhu, W., Yang, Y., Mei, J., Bi, C., et al. (2017). Ss-Rhs1, a secretory Rhs repeat-containing protein, is required for the virulence of Sclerotinia sclerotiorum. Mol. Plant Pathol. 18, 1052-1061. doi: 10.1111/mpp. 12459

Zhang, L. B., Tang, L., Ying, S. H., and Feng, M. G. (2016). Distinct roles of two cytoplasmic thioredoxin reductases $(\operatorname{Trr} 1 / 2)$ in the redox system involving cysteine synthesis and host infection of Beauveria bassiana. Appl. Microbiol. Biotechnol. 100, 10363-10374. doi: 10.1007/s00253-0167688-0

Conflict of Interest Statement: The authors declare that the research was conducted in the absence of any commercial or financial relationships that could be construed as a potential conflict of interest.

Copyright $\odot 2019$ Zhang, Wang, Du, Huang, Fang, Yang, Bi, Qing and Yu. This is an open-access article distributed under the terms of the Creative Commons Attribution License (CC BY). The use, distribution or reproduction in other forums is permitted, provided the original author(s) and the copyright owner(s) are credited and that the original publication in this journal is cited, in accordance with accepted academic practice. No use, distribution or reproduction is permitted which does not comply with these terms. 